\title{
A NEW CHARACTERIZATION FOR $p$-LOCAL BALANCED PROJECTIVE GROUPS
}

\author{
MARK LANE
}

\begin{abstract}
By introducing the notion of a K-nice submodule, we obtain a characterization of $p$-local balanced projectives perfectly analogous to the familiar third axiom of countability characterization of totally projective $p$-groups. We use this new characterization to prove that if a $p$-local group $G$ satisfies the third axiom of countability with respect to nice submodules and has a $\mathrm{K}$-basis, then $G$ is a balanced projective.
\end{abstract}

Balanced projectives were introduced by Warfield in [8] and studied more extensively by him in [9]. The importance of his work is that it provides classification theorems for certain classes of mixed abelian groups. Warfield also introduced the concept of a K-basis which succeeded in isolating the difficulty with the elements of infinite order. Subsequent to these papers, much has been written on valuations and valuated groups which allows one to get a better grasp of the ideas underlying the notion of a K-basis. We refer the reader to [6]. In the present paper we introduce a class of $Z_{p}$-modules that turns out to coincide with the $p$-local balanced projective groups of Warfield. Although our method does not immediately lend itself to a global characterization such as in [9], we do believe that our new point of view will lead to a deeper insight into the structure of balanced projectives.

Let $p$ be a fixed prime, and let $Z_{p}$ denote the ring of integers localized at $p$. All modules in this paper will be assumed to be $Z_{p}$-modules, and we note, of course, that torsion $Z_{p}$-modules are merely abelian $p$-groups. Recall that the height $h_{G}(x)$ of an element $x \in G$ is the ordinal $\alpha$ if $x \in p^{\alpha} G$ but $x \notin p^{\alpha+1} G$, and $h_{G}(x)=\infty$ if $x \in p^{\alpha} G$ for all ordinals $\alpha$. When the context is clear as to the module $G$ in which the heights are being computed, we will write $|x|$ for $h_{G}(x)$. A direct sum $A \oplus B$ of submodules $A$ and $B$ of $G$ is a valuated coproduct if $|a+b|=\min (|a|,|b|)$ for all $a \in A$ and $b \in B$. This notion generalizes in the obvious manner to any number of submodules. Following Richman and Walker in [6], a submodule $F$ is said to be free valuated if there is a direct decomposition $H=\oplus\left\langle x_{i}\right\rangle$, where $o\left(x_{i}\right)=\infty$ for each $i,\left|p^{k} x_{i}\right|=\left|x_{i}\right|+k$ for all $i \in I$ and all nonnegative integers $k$, and the direct decomposition is a valuated coproduct. A useful observation is that $\left|p^{k} x\right|=|x|+k$

Received by the editors July 22, 1984 and, in revised form, March 15, 1985. The contents of this paper represent part of a talk the author gave at a special session of the American Mathematical Society in Abelian Groups held in Tucson, Arizona, April 12-13, 1985. The talk was sponsored by Ross Beaumont. 1980 Mathematics Subject Classification. Primary 20K21, 20K10, $20 \mathrm{~K} 30$.

Ke' words and phrases. Balanced projective groups, K-module. K-nice, third axiom of countability. Ulm invariants, and $h$-invariants. 
whenever $x$ is a nonzero element in a free valuated submodule $F$. In agreement with Hill [3], we say that a submodule $A$ is compatible with a submodule $B$, written $A \| B$, if for any $(a, b) \in A \times B$ there is an $x \in A \cap B$ such that $|a+b| \leqslant|a+x|$. $A$ is a nice submodule of $G$ provided $p^{\alpha}(G / A)=p^{\alpha} G+A / A$ for all ordinals $\alpha$. It is a fundamental and elementary fact that if $A$ is a nice submodule of $G$ and $A \| B$, then the canonical isomorphism $B+A / A \simeq B / A \cap B$ preserves heights as computed in $G / A$ and $G / A \cap B$, respectively.

1. K-nice submodules. A major role in the study of the class of modules that we are to introduce will be played by those $Z_{p}$-modules that we shall designate as " $\mathrm{K}$-modules". Precisely, we call $G$ a $K$-module provided every finitely generated submodule $H$ contains a free valuated submodule $F$ with $H / F$ finite. (From the point of view of valuated groups [6], the finitely generated submodules of $\mathrm{K}$-modules are exactly the finitely presented $p$-local valuated groups, and therefore countable K-modules are just the countable KT-modules studied constructively by Richman in [5]. But as our definition indicates, our main inspiration has been the idea of exploiting the local structure of modules with K-bases in order to develop a classification theory for uncountable modules in the spirit of Hill [2].)

Lemma 1.1 (The Exchange Lemma). If $x$ is a nonzero element of the finitely generated free valuated submodule $F$, then $F$ contains generators $y_{1}, y_{2}, \ldots, y_{m}$ such that both $F=\left\langle y_{1}\right\rangle \oplus\left\langle y_{2}\right\rangle \oplus \cdots \oplus\left\langle y_{m}\right\rangle$ and $F^{\prime}=\langle x\rangle \oplus\left\langle y_{2}\right\rangle \oplus \cdots \oplus\left\langle y_{m}\right\rangle$ are valuated coproducts with $F / F^{\prime}$ finite.

Proof. Suppose $F=\left\langle y_{1}\right\rangle \oplus \cdots \oplus\left\langle y_{m}\right\rangle$ is a valuated coproduct and $x=t_{1} y_{1}+$ $t_{2} y_{2}+\cdots+t_{m} y_{m}$ with $|x|=\left|t_{1} y_{1}\right|$. Then if $F^{\prime}=\langle x\rangle \oplus\left\langle y_{2}\right\rangle \oplus \cdots \oplus\left\langle y_{m}\right\rangle$, it is trivial that $F / F^{\prime}$ is finite, so it remains to show that $\langle x\rangle \oplus\left\langle y_{2}\right\rangle \oplus \cdots \oplus\left\langle y_{m}\right\rangle$ is a valuated coproduct. If $Y=\left\langle y_{2}\right\rangle \oplus \cdots \oplus\left\langle y_{m}\right\rangle$, then it suffices to show that $\langle x\rangle \oplus Y$ is a valuated coproduct. Suppose $s \in Z_{p}$ and $y \in Y$, where $y=s_{2} y_{2}+\cdots+s_{m} y_{m}$; then $|s x+y|=\left|s t_{1} y_{1}+\left(s t_{2}+s_{2}\right) y_{2}+\cdots+\left(s t_{m}+s_{m}\right) y_{m}\right| \leqslant\left|s t_{1} y_{1}\right|=|s x|$ since $\left\langle y_{1}\right\rangle \oplus\left\langle y_{2}\right\rangle \oplus \cdots \oplus\left\langle y_{m}\right\rangle$ is a valuated coproduct. Hence $\langle x\rangle \oplus\left\langle y_{2}\right\rangle$ $\oplus \cdots \oplus\left\langle y_{m}\right\rangle$ is also a valuated coproduct.

Lemma 1.2. If $A / C$ is torsion and if $C \oplus F$ is a valuated coproduct with $F$ a free valuated submodule, then $A \oplus F$ is also a valuated coproduct.

Proof. First observe that $A \cap F=0$ since $A / C$ is torsion and $F$ is torsion-free. Assume, by way of contradiction, that $|a+f|>|f|$ for some $a \in A$ and some $f \in F$. Choosing a nonnegative integer $k$ such that $p^{k} a \in C$, we are led to the absurdity $|f|+k<|a+f|+k \leqslant\left|p^{k} a+p^{k} f\right| \leqslant\left|p^{k} f\right|=|f|+k$.

Corollary 1.3. If $A$ is a submodule of the finitely generated submodule $H$ of the $K$-module $G$, then $H$ contains as a submodule a free valuated module $B$ with $H / B$ finite, $B=(A \cap B) \oplus C$ a valuated coproduct, and $A \oplus C$ a valuated coproduct .

Proof. Since $H$ is a K-module, $H$ contains a free valuated module $F$ with $H / F$ finite. A succession of applications of Lemma 1.1 yields a valuated coproduct $B=\left\langle x_{1}\right\rangle \oplus \cdots \oplus\left\langle x_{n}\right\rangle \oplus C$, where $F / B$ is finite, the $x_{i}$ 's are in $A \cap F$, and $C$ is a 
free valuated submodule with $A \cap C=0$. It follows then that $A \cap B=$ $\left\langle x_{1}, x_{2}, \ldots, x_{n}\right\rangle$ and, by Lemma 1.2 , that $A \oplus C$ is a valuated coproduct since $A / A \cap B \simeq B+A / B$ is finite because $H / B$ is.

Lemma 1.4. If $A$ is a finitely generated nice submodule of the $K$-module $G$, then $G / A$ is also a $\mathrm{K}$-module.

Proof. Suppose $H / A$ is a finitely generated submodule of $G / A$. Then $H$ is itself necessarily a finitely generated submodule of $G$, and hence $H$ contains a free valuated submodule $B$ as in Corollary 1.3. It is now easily verified that $A \oplus C$ being a valuated coproduct implies $A \| B$. Thus, as noted in the introduction, the canonical isomorphisms $B+A / A \simeq B / A \cap B \simeq C$ preserve heights in the corresponding quotients of $G$. Therefore $B+A / A$ is a free valuated submodule of $G / A$ and the desired conclusion follows since clearly $H / B+A$ is finite.

Definition 1.5. A submodule $A$ of $G$ is $K$-nice if $A$ is nice in $G$ and $G / A$ is a K-module.

Proposition 1.6. If $A$ is $K$-nice in $G$, and $B / A$ is $K$-nice in $G / A$, then $B$ is $K$-nice in $G$.

Proof. That $B$ is at least nice in $G$ under these circumstances is well known. Moreover, the canonical isomorphism $G / B \simeq G / A / B / A$ implies that $G / B$ is a K-module.

THEOREM 1.7. Finitely generated submodules of $K$-modules are $K$-nice.

Proof. Let $A$ be a finitely generated submodule of the K-module $G$. By Lemma 1.4 and Definition 1.5 , it suffices to show that $A$ is nice in $G$. Pick a free valuated submodule $F$ with $A / F$ finite. Since niceness is preserved under finite extensions, we need only prove that $F$ is nice in $G$. Using induction on the rank of $F$ and Lemma 1.4 again, we see that it is enough to consider the special case $F=\langle x\rangle$, where $o(x)=\infty$ and $\left|p^{k} x\right|=|x|+k$ for all nonnegative integers $k$. Now let $g$ be an arbitrary element of $G$ and recall that we need only argue that the coset $g+\langle x\rangle$ contains an element of maximal height. Since $B=\langle g, x\rangle$ is a finitel y generated submodule of $G$, there is a free valuated submodule $C$ with $B / C$ finite. But then the Exchange Lemma 1.1 yields a valuated coproduct $C^{\prime}=\left\langle x^{\prime}\right\rangle \oplus D$, where $x^{\prime}$ is a nonzero multiple of $x$ and $C / C^{\prime}$ is finite. Thus we can write $p^{k} g=a x+y$, where $k$ is a nonnegative integer, $a \in Z_{p}$, and $y \in D$. Since $\langle x\rangle \oplus\langle y\rangle$ is a valuated coproduct by Lemma 1.2, the argument is completed precisely as in the proof of Lemma 8.1 in [4].

Corollary 1.8. If $A$ is $K$-nice in $G$ and $B / A$ is finitely generated, then $B$ is $K$-nice in $G$.

2. The isomorphism theorem. The results in the preceding section have shown how $\mathrm{K}$-nice submodules in $p$-local modules generalize nice subgroups in $p$-groups. This observation suggests that a generalization of Ulm's theorem for totally projective 
$p$-groups is possible. We now introduce our new class of modules which will make such a generalization possible.

Definition 2.1. A $Z_{p}$-module $G$ is said to satisfy the third axiom of countability with respect to $K$-nice submodules provided there is a family $\mathscr{C}$ of $\mathrm{K}$-nice submodules of $G$ satisfying the following conditions:

(0) $0 \in \mathscr{C}$,

(1) $\sum N_{i} \in \mathscr{C}$ provided each $N_{i} \in \mathscr{C}$, and

(2) if $X$ is a countable subset of $G$, then there is a countable $N \in \mathscr{C}$ with $X \subseteq N$.

We follow Warfield in [8] and [9] and define the invariants

$$
h(0, G)=\operatorname{dim}[G / p G+T],
$$

where $T$ is the torsion part of $G$, and $h(\lambda, G)=h\left(0, p^{\lambda} G\right)$ when $\lambda$ is a limit ordinal. It is convenient for us to consider 0 as a limit ordinal, and we will denote $T_{\lambda}$ as the torsion part of $p^{\lambda} G$. Recall that the Ulm invariants of $G$ are, for each ordinal $\alpha$, the cardinal numbers $f_{G}(\alpha)=\operatorname{dim}\left(p^{\alpha} G[p] / p^{\alpha+1} G[p]\right)$. In addition, we define $f_{G}(\infty)=$ $\operatorname{dim} p^{\infty} G[p]$ and $h(\infty, G)=\operatorname{dim}\left(p^{\infty} G \otimes Q\right)$, where $Q$ is the quotient field of $Z_{p}$. Since it is well known that $f_{G}(\infty)$ and $h(\infty, G)$ classify the divisible $Z_{p}$-modules, divisible modules do satisfy the third axiom of countability with respect to K-nice submodules (when agreeing, of course, that $\infty+n=\infty$ for all integers $n$ ), and the divisible part of $G$ can be factored out and disposed of at the beginning, we may as well assume that all modules to follow are reduced. We now state the main theorem of this section.

THEOREM 2.2. Two $Z_{p}$-modules which satisfy the third axiom of countability with respect to $K$-nice submodules are isomorphic if and only if they have the same Ulm and h-invariants.

The technical aspects of proving this theorem involve relative invariants. Recall that for a nice subgroup $A$ in the $p$-group $G$, Hill in [2] developed the relative Ulm invariants with respect to $A$ which succeeded in isolating the problem of extending isomorphisms. We use Walker's definition in [7]. Define $A(\alpha)=\left(p^{\alpha+1} G+A\right) \cap$ $p^{\alpha} G[p]$ and the relative Ulm invariants with respect to $A$ to be $f_{G}(\alpha, A)=$ $\operatorname{dim}\left[p^{\alpha} G[p] / A(\alpha)\right]$ for each ordinal $\alpha$. Similar to Hill's approach, in our work we want to use the relative Ulm invariants and define the relative h-invariants with respect to $A$. Define $A_{\lambda}=\left(p^{\lambda+1} G+A+T_{\lambda}\right) \cap p^{\lambda} G$ for all ordinals $\lambda$. Since for all $\lambda$, the map from $p^{\lambda} G$ to $p^{\lambda+1} G$ given by $x \mapsto p x$ carries $A_{\lambda}$ into $A_{\lambda+1}$, there is a direct sequence of maps

$$
p^{\lambda} G / A_{\lambda} \rightarrow p^{\lambda+1} G / A_{\lambda+1} \rightarrow \cdots \rightarrow p^{\lambda+n} G / A_{\lambda+n} \rightarrow \cdots,
$$

where $\lambda$ is a limit ordinal. We will write $H_{G}(\lambda, A)$ for the vector space $\lim _{\rightarrow} p^{\lambda+n} G / A_{\lambda+n}$ and $\delta_{\lambda}^{A}$ for the canonical map $p^{\lambda} G \rightarrow H_{G}(\lambda, A)$. We now define the relative $h$ invariant with respect to $A$ as $h_{G}(\lambda, A)=\operatorname{dim} H_{G}(\lambda, A)$. Since "direct limits of exact sequences are exact", if $A \subseteq B$, we have the following short exact sequence:

$$
0 \rightarrow[B / A]_{\lambda} \rightarrow H_{G}(\lambda, A) \rightarrow H_{G}(\lambda, B) \rightarrow 0,
$$


where $[B / A]_{\lambda}$ is defined to be the direct limit $\lim _{\rightarrow} B_{\lambda+n} / A_{\lambda+n}$. Since

$$
p^{\lambda} G / p^{\lambda+1} G+T_{\lambda} \simeq p^{\lambda+n} G / p^{\lambda+n+1} G+T_{\lambda+n} \quad \text { for every } n<\omega,
$$

it follows that $h_{G}(\lambda, 0)=h(\lambda, G)$ for any limit ordinal $\lambda$.

Lemma 2.3. Let $x \in p^{\lambda} G$. Then $\delta_{\lambda}^{A}(x) \neq 0$ if and only if $A \oplus\langle x\rangle$ is a valuated coproduct and $\left|p^{n} x\right|=\lambda+n$ for all $n<\omega$.

Proof. Assume that $\delta_{\lambda}^{A}(x) \neq 0$; that is, $p^{n} x \notin A_{\lambda+n}$ for all $n<\omega$. First observe that $p^{n} x \notin A_{\lambda+n}$ implies that $\left|p^{n} x\right| \leqslant \lambda+n$. On the other hand, if $A \oplus\langle x\rangle$ were not a valuated coproduct, then we would have $\left|a+p^{n} x\right|>\left|p^{n} x\right|$ for some $a \in A$ and some $n<\omega$-contradicting $p^{n} x \notin A_{\lambda+n}$.

Conversely suppose $A \oplus\langle x\rangle$ is a valuated coproduct and $\left|p^{n} x\right|=\lambda+n$ for all $n<\omega$. If $p^{n} x \in A_{\lambda+n}$ for some integer $n$, then write $p^{n} x=g+a+t$ where $g \in p^{\lambda+n+1} G, a \in A$, and $t \in T_{\lambda+n}$. There exists a nonnegative integer $k$ such that $p^{k} t=0$, and this implies that $\lambda+n+k+1 \leqslant\left|p^{n+k} x-p^{k} a\right|$ which contradicts the hypotheses. So $p^{n} x \notin A_{\lambda+n}$ for each integer $n$, and we conclude $\delta_{\lambda}^{A}(x) \neq 0$.

Lemma 2.4. Let $\pi: A \rightarrow A^{\prime}$ be a height preserving isomorphism where $A$ and $A^{\prime}$ are $K$-nice submodules of $G$ and $G^{\prime}$, respectively. Suppose also that for all ordinals $\alpha$ and all limit ordinals $\lambda$ there exist isomorphisms

$$
\mu_{\alpha}: p^{\alpha} G[p] / A(\alpha) \rightarrow p^{\alpha} G^{\prime}[p] / A^{\prime}(\alpha) \quad \text { and } \quad \sigma_{\lambda}: H_{G}(\lambda, A) \rightarrow H_{G^{\prime}}\left(\lambda, A^{\prime}\right) .
$$

Then if $B$ is a submodule of $G$ such that $B / A$ is finitely generated, there exists a height preserving isomorphism $\theta: B \rightarrow B^{\prime}$, where $B^{\prime}$ is a submodule of $G^{\prime}$ and, for all $\alpha$ and $\lambda, \mu_{\alpha}$ and $\sigma_{\lambda}$ induce isomorphisms of $B(\alpha) / A(\alpha)$ onto $B^{\prime}(\alpha) / A^{\prime}(\alpha)$ and of $[B / A]_{\lambda}$ onto $\left[B^{\prime} / A^{\prime}\right]_{\lambda}$. Furthermore, $B$ and $B^{\prime}$ are $K$-nice in $G$ and $G^{\prime}$, respectively, and for all $\alpha$ and all limit ordinals $\lambda, f_{G}(\alpha, B)=f_{G^{\prime}}\left(\alpha, B^{\prime}\right)$ and $h_{G}(\lambda, B)=h_{G^{\prime}}\left(\lambda, B^{\prime}\right)$.

Proof. We may assume $B=\langle A, x\rangle$, where $x \in G \backslash A$. When $x+A$ has finite order, we proceed as Walker does in [7]. In this case it is clear that $[B / A]_{\lambda}$ and $\left[B^{\prime} / A^{\prime}\right]_{\lambda}$ are both trivial. Suppose $x+A$ has infinite order. Since $A$ is K-nice in $G$, there is some $x^{\prime} \in G$ and some $k<\omega$ such that $A \oplus\left\langle x^{\prime}\right\rangle$ is a valuated coproduct, $\left|p^{\prime \prime} x^{\prime}\right|=\left|x^{\prime}\right|+n$ for all $n<\omega$, and $p^{k} x \in A \oplus\left\langle x^{\prime}\right\rangle$. In light of the first case, we may replace $x$ by $x^{\prime}$. We may also assume, without loss of generality, that the height of $x$ is a limit ordinal $\lambda$; for otherwise if $|x|=\lambda+n$ for $n \neq 0$, then $x=p^{n} x_{1}$ where $\left|x_{1}\right|=\lambda$, so if we replace $x$ by $x_{1}$, we see that $x \in A \oplus\left\langle x_{1}\right\rangle$. By Lemma 2.3, $\delta_{\lambda}^{A}(x) \neq 0$, and so $\sigma_{\lambda}\left(\delta_{\lambda}^{A}(x)\right) \neq 0$. This implies that there is some $y \in p^{\lambda} G^{\prime}$ such that $0 \neq \delta_{\lambda}^{A^{\prime}}(y)=\sigma_{\lambda}\left(\delta_{\lambda}^{A}(x)\right)$. Again by Lemma 2.3, $A^{\prime} \oplus\langle y\rangle$ is a valuated coproduct and for all $n<\omega,\left|p^{n} y\right|=\lambda+n$. Now we define $\theta$ by $\theta(a+s x)=\pi(a)+s y$ for all $a \in A$ and $s \in Z_{p}$. When $\beta$ is a limit ordinal not equal to $\lambda, B_{\beta}=A_{\beta}$, and when $\beta=\lambda,[B / A]_{\lambda}$ is cyclic of order $p$. Hence, $\sigma_{\beta}$ induces an isomorphism of $[B / A]_{\beta}$ onto $\left[B^{\prime} / A^{\prime}\right]_{\beta}$ for all limit ordinals $\beta$ as desired. It is also clear that in this case $A(\alpha)=B(\alpha)$ for all ordinals $\alpha$, so $\mu_{\alpha}$ trivially induces an isomorphism as 
desired. Because $B / A$ and $B^{\prime} / A^{\prime}$ are finitely generated and $A$ and $A^{\prime}$ are $\mathrm{K}$-nice, it follows by Corollary 1.8 that $B$ and $B^{\prime}$ are K-nice in $G$ and $G^{\prime}$, respectively. That $f_{i}(\alpha, B)=f_{G^{\prime}}\left(\alpha, B^{\prime}\right)$ is a consequence of the induced isomorphism from $\mu_{\alpha}$, and that $h_{G}(\lambda, B)=h_{G^{\prime}}\left(\lambda, B^{\prime}\right)$ is a consequence of the induced isomorphism from $\sigma_{\lambda}$ and the exact sequence $(\dagger)$.

Armed with Lemma 2.4 and the straightforward observation that $[B / A]_{\lambda}=$ $\cup\left[B_{n} / A\right]_{\lambda}$ when $B=\bigcup B_{n}$, an ascending union, one adapts Walker's argument in [7] without difficulty to prove the following generalized version of Theorem 2.2.

THEOREM 2.5. If $G / A$ and $G^{\prime} / A^{\prime}$ satisfy the third axiom of countability with respect to $K$-nice submodules with $A$ and $A^{\prime} K$-nice in $G$ and $G^{\prime}$, respectively, and if $f_{G}(\alpha, A)=f_{G^{\prime}}\left(\alpha, A^{\prime}\right)$ for each ordinal $\alpha$ and $h_{G}(\lambda, A)=\dot{n}_{G^{\prime}}\left(\lambda, A^{\prime}\right)$ for all limit ordinals $\lambda$, then any height preserving isomorphism $A \rightarrow A^{\prime}$ extends to an isomorphism $G \rightarrow G^{\prime}$.

3. The $p$-local balanced projectives. In this section we prove that our modules satisfying the third axiom of countability with respect to K-nice submodules are precisely the $p$-local balanced projectives, and we close by answering a long standing problem posed by Warfield. In agreement with Warfield [9], a module $M$ is a $\lambda$-elementary $K T$-module if $p^{\lambda} M \simeq Z_{p}$ and $M / p^{\lambda} M$ is torsion and totally projective. We recall that a $p$-local group is a balanced projective if it is a direct sum of a divisible module, a torsion totally projective module, and $\lambda$-elementary KT-modules for various limit ordinals $\lambda$.

THEOREM 3.1. The class $\mathscr{C}$ of all modules which satisfy the third axiom of countability with respect to $K$-nice submodules coincides with the class $\mathscr{K}$ of all p-local balanced projectives.

Proof. It was observed that all divisible modules are in $\mathscr{C}$, and it is clear that torsion totally projective modules are also in $\mathscr{C}$. Consider a $\lambda$-elementary KT-module $M$. Since $p^{\lambda} M \simeq Z_{p}$ and $M / p^{\lambda} M$ is torsion totally projective, then $p^{\lambda} M$ and $M / p^{\lambda} M$ are K-modules with $p^{\lambda} M$ countable and $\mathrm{K}$-nice in $M$. $M$ will be in $\mathscr{C}$ if $M$ is a $\mathrm{K}$-module. But this is obviously so since if $H$ is a finitely generated submodule of $M$, then $H / F$ is finite, where either $F=0$ or else $F=p^{\lambda+n} M \simeq Z_{p}$ for some $n<\omega$.

Now $\mathscr{K} \subseteq \mathscr{C}$ if direct sums of modules in $\mathscr{C}$ are in $\mathscr{C}$. By the well-known proof of Lemma 81.5 in [1], it is enough to show that direct sums of K-modules are $\mathrm{K}$-modules. Suppose $G=\oplus_{i \in I} G_{i}$, where for each $i \in I, G_{i}$ is a K-module, and let $H$ be a finitely generated submodule in $G$. Since $H$ is finitely generated, $H$ is contained in the sum of finitely many of the $G_{i}$ 's. Thus we only need prove the result when $I$ is finite, and hence, by a trivial induction, when $I=\{1,2\}$. In this case, $H \subseteq H_{1} \oplus H_{2}$, where $H_{1}$ and $H_{2}$ are finitely generated submodules of $G_{1}$ and $G_{2}$, respectively. Then there exists a free valuated module $F=F_{1} \oplus F_{2}$ where each $H_{i} / F_{i}$ is finite. As in the proof of $1.3, H_{1} \oplus H_{2}$ contains a free valuated submodule $B$ with $\left(H_{1} \oplus H_{2}\right) / B$ finite and $B \cap H$ free valuated. Hence $G$ is a $\mathrm{K}$-module since $H / H \cap B$ is clearly finite. 
Now we refer the reader to Corollary 4.5 in [8]; since $\mathscr{C}$ is classified by the Ulm and $h$-invariants, and $\mathscr{K} \subseteq \mathscr{C}$, we conclude that $\mathscr{C}$ coincides with $\mathscr{K}$.

We pointed out in the introduction that our new characterization should lead to a deeper insight into the structure of the balanced projectives. Indeed the third axiom of countability characterization of these modules should open up the possibility for generalizing various results heretofore known only for totally projective $p$-groups. To illustrate the power of our characterization, we will close this paper with a solution to a problem posed by Warfield in [9]: if a $p$-local group $G$ has a K-basis and satisfies the third axiom of countability with respect to nice submodules, is $G$ necessarily a balanced projective? It is worth remarking that the notion of a K-basis was fundamental in the evolution of the definitions of $\mathrm{K}$-module and $\mathrm{K}$-nice. We follow Warfield in [9] and call a set $X$ a $K$-basis provided $\langle X\rangle$ is a free valuated module in $G$ and $G /\langle X\rangle$ is torsion.

THEOREM 3.2. If $G$ is a p-local group that satisfies the third axiom of countability with respect to nice submodules and $G$ has a $K$-basis $X$, then $G$ is a balanced projective.

Proof. We aim to show $G$ satisfies the third axiom of countability with respect to $\mathrm{K}$-nice submodules. To this end, if $\mathscr{C}$ is the collection of nice submodules satisfying the third axiom of countability, we will show that $\mathscr{C}$ contains a subcollection, $\mathscr{C}^{\prime}$, of K-nice submodules such that $\mathscr{C}^{\prime}$ also satisfies the third axiom of countability. First note that, as the proof of Corollary 1.3 shows, any module that has a K-basis is a K-module. For $N \in \mathscr{C}$, let $X_{N}=X \cap N$ and $Y_{N}=X \backslash X_{N}$, and let $\mathscr{C}^{\prime}=\{N \in$ $\mathscr{C} \mid N /\left\langle X_{N}\right\rangle$ is torsion $\}$. Suppose $N \in \mathscr{C}^{\prime}$. Since $N /\left\langle X_{N}\right\rangle$ is torsion and $\left\langle X_{N}\right\rangle \cap$ $\left\langle Y_{N}\right\rangle=0$, it follows that $N \cap\left\langle Y_{N}\right\rangle=0$. Also, since $\left\langle X_{N}\right\rangle \oplus\left\langle Y_{N}\right\rangle$ is a valuated coproduct, $N \oplus\left\langle Y_{N}\right\rangle$ is a valuated coproduct by Lemma 1.2. Since $N$ is nice and $N \oplus\left\langle Y_{N}\right\rangle$ is a valuated coproduct, it follows that $\left(N \oplus\left\langle Y_{N}\right\rangle\right) / N$ is a free valuated module. So $G / N$ has the K-basis $\left\{y+N \mid y \in Y_{N}\right\}$, and thus $G / N$ is a K-module.

It is easy to see that the sum of an arbitrary number of modules in $\mathscr{C}^{\prime}$ is in $\mathscr{C}^{\prime}$, so suppose that $C$ is a countable subset of $G$. There exists a countable $A_{1} \in \mathscr{C}$ with $C \subseteq A_{1}$. If $A_{1} \in \mathscr{C} \backslash \mathscr{C}^{\prime}$, then there will at least be a countable submodule $B_{1}$ of $G$ containing $A_{1}$ such that $B_{1} /\left\langle X_{B_{1}}\right\rangle$ is torsion. So there exists a countable $A_{2} \in \mathscr{C}$ such that $B_{1} \subseteq A_{2}$. Continuing in this manner, $N=\bigcup A_{n}=\bigcup B_{n}$ is a countable member of $\mathscr{C}^{\prime}$ containing $C$. Hence $\mathscr{C}^{\prime}$ satisfies the third axiom of countability with respect to $\mathrm{K}$-nice submodules, and $G$ is a balanced projective by 3.1 .

Addendum (March, 1985). A couple of months after this paper was submitted, P. Hill and C. Megibben obtained a third axiom of countability characterization of the more general class of $p$-local Warfield modules and also extended Theorem 3.2, with "K-basis" replaced by "decomposition basis", to those modules. Their work, however, requires several new ideas that have not arisen in our treatment of $p$-local balanced projectives. In particular, their generalization of our notion of a $\mathrm{K}$-nice subgroup is not susceptible to a brief formulation in terms of familiar concepts such as free valuated groups and nice submodules. 


\section{REFERENCES}

1. L. Fuchs, Infinite abelian groups, vol. II, Academic Press, New York, 1973.

2. P. Hill, On the classification of abelian groups, photocopied manuscript.

3. __ Isotype subgroups of totally projective groups, Lecture Notes in Math., Vol. 874, SpringerVerlag. Berlin and New York, 1981, pp. 305-321.

4. R. Hunter and F. Richman, Global Warfield groups, Trans. Amer. Math. Soc. 266 (1981), 555-572.

5. F. Richman, The constructive theory of KT-modules, Pacific J. Math. 61 (1975), 263-274.

6. F. Richman and E. A. Walker, Valuated groups, J. Algebra 56 (1979), 145-167.

7. E. A. Walker, Ulm's theorem for totally projective groups, Proc. Amer. Math. Soc. 37 (1973), 387-392.

8. R. Warfield, A classification theorem for abelian p-groups, Trans. Amer. Math. Soc. 210 (1975), 149-168.

9. Classification theory of abelian groups. I: Balanced projectives, Trans. Amer. Math. Soc. 222 (1976). 33-63.

Department of Mathematics, Vanderbilt University, NashVille, TenNessee 37235

Current address: Department of Mathematics, Auburn University, Auburn, Alabama 36849-3501 